\title{
Clinical Effect and Effective Rate of Laparoscopic Cholecystectomy for Gallstones Complicated with Gallbladder Polyps
}

\author{
Xiaoming Deng ${ }^{1}$, Jinjun Jiang* \\ ${ }^{1}$ Jiayuguan Jiugang Hospital of Gansu Province,735100, Jiayuguan City, China \\ *Linze County People's Hospital ,734200, Linze, China
}

\begin{abstract}
This paperA total of 62 patients with gallstones and gallbladder polyps were selected from May 2019 to May 2020, who were divided into the observation group ( $\mathrm{n}=31$, laparoscopic cholecystectomy) and the control group ( $n=31$, open cholecystectomy) in a 1:1 ratio. The clinical indicators, clinical efficacy, level of pain and complication rate of the two groups were recorded and compared. Results Indicators such as the operation duration $(38.64 \pm 14.42 \mathrm{~min})$, blood loss $(30.42 \pm 8.21 \mathrm{ml})$, length of stay $(4.71 \pm 1.82 \mathrm{~d})$, first anal exhaust $(21.82 \pm 6.65 \mathrm{~min})$, drainage volume $(72.02 \pm 4.21 \mathrm{ml})$, length of incision and the time for the recovery of gastrointestinal functions in the observation group were better than the control group $(\mathrm{P}<0.05)$. The clinical efficacy of the observation group (96.77\%) was higher than that of the control group (80.65\%), with statistical value $=4.0260(\mathrm{P}<0.05)$. The level of pain of the observation group was lower than that of control group $(\mathrm{P}<0.05)$, while the complication rate in the observation group $(3.22 \%)$ was also lower than that of the control group $(22.58 \%)(\mathrm{P}<0.05)$. Laparoscopic cholecystectomy is an effective treatment of gallstones complicated with gallbladder polyps, which can alleviate pain and improve the prognosis, and is thus worthy of promotion.
\end{abstract}

\section{Introduction}

Gallstones and gallbladder polyps are common abdominal diseases. Clinically, gallstones are closely related to obesity and diet, while gallbladder polyps have to do with gallstones and abnormal cholesterol metabolism, accompanied by abdominal pain, discomfort and other symptoms after onset. The combination of the two will accelerate the progress of the disease. In severe cases, it could progress to secondary cholangitis, endangering patients' lives ${ }^{[1]}$. Open cholecystectomy is a common treatment for gallstones with gallbladder polyps. However, major trauma, serious blood loss, and complications such as indigestion and reflux gastritis make it a non-ideal option, demanding a more secure and effective solution ${ }^{[2]}$. With the advance of medical technologies in recent years, laparoscopy has been widely used in the treatment of abdominal diseases due to smaller incision and less bleeding. This treatment provides doctors with visual vision during the surgery, helping them locate pathological tissues quickly and shorten the operation duration. This paper selected 62 patients from May 2019 to May 2020 to clarify the efficacy of the treatment against gallstones and gallbladder polyps.

\section{Material and Method}

\subsection{General information}

Sixty-two patients with gallstones and gallbladder polyps from May 2019 to May 2020 were included in the study and were divided into two groups in the 1:1 ratio. The observation group consisted of 31 patients, with male/female $=18 / 13$ and an average age of $(43.43 \pm 3.24)$ years old. The mean time of onset was $(4.91 \pm 0.42) \mathrm{h}$; The mean stone diameter was $(0.82 \pm 0.11) \mathrm{cm}$. There were 31 patients in the control group, with male/female $=17 / 14$ and an average age of $(43.41 \pm 3.05)$ years. The mean time of onset was $(4.94 \pm 0.43) \mathrm{h}$; The mean diameter of stones was $(0.81 \pm 0.12) \mathrm{cm}(\mathrm{P}>0.05)$.

\subsubsection{Inclusion criteria}

(1) Diagnosed by abdominal CT and B-ultrasound; (2) In a good mental state; (3) No contraindication of operation; (4) Voluntarily join in and sign the "informed consent form"; (5) Without blood system diseases; (6) No immune system disease; (7) No abdominal surgery recently ${ }^{[3]}$.

\footnotetext{
* Corresponding author: jsvc3634@163.com
} 


\subsubsection{Exclusion criteria}

(1) Mental and psychological diseases; (2) Abdominal malignant tumor; (3) Patients in the state of unconsciousness; (4) Organ dysfunction; (5) Patients with contraindications; (6) Voluntary withdrawal from this study; (7) Loss of clinical data; (8) Cardiovascular and cerebrovascular diseases; (9) Cardiopulmonary insufficiency

\subsection{Method}

2.2.1 The control group was treated with open cholecystectomy: The patients were given general anesthesia with endotracheal intubation, and then assisted to lie down in a supine position with slight left inclination, with their heads high and feet low. After routine disinfection and surgical drape, the operation was performed. A $5 \mathrm{~cm}$ incision was made from the right lower abdomen, where the abdominal wall tissues were cut open slowly. After the cystic duct and gallbladder artery were identified, they were ligated. The gallbladder was removed and the stones were taken out before the incision was sutured.

2.2.2 The observation group was treated with laparoscopic cholecystectomy. Patients with anesthesia and lying position were selected as the control group. After performing anesthesia, an incision of $0.5-1.0 \mathrm{~cm}$ was created below the navel, serving as the observation hole. Another incision of $0.5-1.0 \mathrm{~cm}$ was created below the xiphisternum, serving as the operation hole. And an incision of $0.5-1.0 \mathrm{~cm}$ was created at the right midclavicular line and the right anterior axillary line below the right costal margin, serving as the auxiliary operation hole. From the observation hole, a catheter was inserted to create an artificial pneumoperitoneum. The exploration scope and surgical instruments were inserted from the operation hole, then the gallbladder artery was cut off and ligated. After that, the common bile duct was pulled out based on the position of the cystic duct, with the proximal end being clamped (to prevent stones from sliding into the common bile duct during operation). Then the gallbladder was removed. The $\mathrm{CO} 2$ was discharged after the surgery was completed, and the pneumoperitoneum was closed.

Precautions: After the surgery, the drainage tube was retained, and the patients were instructed to take antibiotics orally for 3-5 days until the examination results showed that the blood routine and vital signs had returned to normal.

\subsection{Observation indicators}

(1) Clinical indicators: Operation duration, blood loss, length of stay, first anal exhaust, drainage volume, incision length, recovery of gastrointestinal function, etc.

(2) Based on the clearance rate, the clinical effect was evaluated and divided into the following categories: complete clearance under B-ultrasound (significantly effective), partial clearance under B-ultrasound (effective), and below standard (ineffective) ${ }^{[4]}$.

(3) Complications such as hepatobiliary injury, incision infection, bile leakage and abdominal adhesion were found.

(4) Evaluate the level of pain at $12 \mathrm{~h}, 24 \mathrm{~h}$ and $48 \mathrm{~h}$ after the surgery with VAS scale. The score was 0-10. The higher the score, the higher the level of pain.

\subsection{Statistics}

SPSS22.0 was used to analyze the measurement (expressed in $\bar{x}_{ \pm \mathrm{S}}$ and $\mathrm{T}$ test) and counting data (expressed in $\mathrm{n}, \%$, and $\chi 2$ test). When $\mathrm{P}<0.05$, it was considered statistically significant.

\section{Result}

\subsection{Statistics of indicators for the two groups}

Compared with the control group, seven indicators including the operation duration, blood loss, length of stay, first anal exhaust, drainage volume, length of incision and the time for the recovery of gastrointestinal functions were significantly better $(\mathrm{P}<0.05)$, as shown in Table 1.

Table 1: Clinical Indicators $(\bar{x} \pm s)$

\begin{tabular}{ccccc}
\hline Group & $\begin{array}{c}\text { Observation } \\
\text { Group } \\
(\mathrm{n}=31)\end{array}$ & $\begin{array}{c}\text { Control } \\
\text { Group } \\
(\mathrm{n}=31)\end{array}$ & $\mathrm{t}$ & \\
\hline $\begin{array}{c}\text { Operation } \\
\text { Duration (min) }\end{array}$ & $38.64 \pm 14.42$ & $70.82 \pm 12.42$ & 9.4145 & 0.0000 \\
Blood loss (ml) & $30.42 \pm 8.21$ & $71.54 \pm 12.28$ & 15.4990 & 0.0000 \\
Length of stay & $4.71 \pm 1.82$ & $7.64 \pm 2.11$ & 5.8545 & 0.0000 \\
(d) & & & & \\
First anal & $21.82 \pm 6.65$ & $34.35 \pm 5.91$ & 7.8416 & 0.0000 \\
exhaust (min) & & & & \\
Drainage & $72.02 \pm 4.21$ & $76.24 \pm 4.81$ & 3.6757 & 0.0005 \\
volume (ml) & & & & \\
Length of & $1.22 \pm 0.21$ & $2.98 \pm 0.31$ & 26.1710 & 0.0000 \\
$\begin{array}{c}\text { incision (cm) } \\
\text { Time for the } \\
\text { recovery of } \\
\text { gastrointestinal } \\
\text { functions ( } \mathrm{h})\end{array}$ & $18.52 \pm 4.62$ & $25.94 \pm 3.52$ & 7.1129 & 0.0000 \\
\hline
\end{tabular}

\subsection{Comparison of clinical effects of the two groups}

Compared with the clinical effect of $80.65 \%$ of the control group, the clinical effect of the observation group 
(96.77\%) was significantly higher $(\mathrm{P}<0.05)$, as shown in Table 2.

Table 2: Clinical Effect [n (\%)]

\begin{tabular}{ccccc}
\hline Group & $\begin{array}{c}\text { Significantly } \\
\text { Effective }\end{array}$ & Effective & Ineffective & $\begin{array}{c}\text { Clinical } \\
\text { Effect }\end{array}$ \\
\hline Observation & $16(51.61)$ & $14(45.16)$ & $1(3.23)$ & $96.77 \%$ \\
Group & & & & $(30 / 31)$ \\
$(\mathrm{n}=31)$ & & & & \\
Control & $15(48.39)$ & $10(32.26)$ & $6(19.35)$ & $80.65 \%$ \\
Group & & & & $(25 / 31)$ \\
$(\mathrm{n}=31)$ & & & &
\end{tabular}

$\begin{array}{lllll}x^{2} & -- & -- & -- & 4.0260\end{array}$

\subsection{Comparison of complication rate of the two groups}

Compared with the complication rate of $22.58 \%$ of the control group, the number was significantly lower in the observation group $(3.22 \%)(\mathrm{P}<0.05)$, as shown in Table 3.

Table 3: Complication Rate [n (\%)]

\begin{tabular}{cccccc}
\hline Group & $\begin{array}{c}\text { Intrahepatic bile } \\
\text { duct injury }\end{array}$ & $\begin{array}{c}\text { Incision } \\
\text { infection }\end{array}$ & Bile leakage & $\begin{array}{c}\text { Abdominal } \\
\text { adhesion }\end{array}$ & Total \\
\hline $\begin{array}{c}\text { Observation Group } \\
(\mathrm{n}=31)\end{array}$ & $1(3.23)$ & $0(0.00)$ & $0(0.00)$ & $0(0.00)$ & $3.22 \%(1 / 31)$ \\
$\begin{array}{c}\text { Control Group } \\
(\mathrm{n}=31)\end{array}$ & $3(9.68)$ & $1(3.23)$ & $2(6.45)$ & $1(3.23)$ & $22.58 \%(7 / 31)$ \\
$x^{2}$ & -- & -- & -- & -- & 5.1667 \\
$P$ & -- & -- & -- & -- & 0.0230 \\
\hline
\end{tabular}

\subsection{Comparison of the level of pain of the two groups}

Compared with the control group, the level of pain of the observation group was significantly lower, scored at $3.64 \pm 0.52,2.84 \pm 0.34$ and $2.02 \pm 0.14$ points $12 \mathrm{~h}, 24 \mathrm{~h}$ and $28 \mathrm{~h}$ after the surgery $(\mathrm{P}<0.05)$, as shown in Table 4 .

Table 4: Level of Pain $(\bar{x} \pm s$, point)

\begin{tabular}{cccc}
\hline Group & $\begin{array}{c}12 \mathrm{~h} \text { after } \\
\text { surgery }\end{array}$ & 24h after surgery & $\begin{array}{c}48 \mathrm{~h} \\
\text { surgery }\end{array}$ \\
\hline $\begin{array}{c}\text { Observation } \\
\text { Group }\end{array}$ & $3.64 \pm 0.52$ & $2.84 \pm 0.34$ & $2.02 \pm 0.14$ \\
$(\mathrm{n}=31)$ & & \\
Control & $5.92 \pm 0.42$ & $4.22 \pm 0.38$ & $3.42 \pm 0.31$ \\
Group & & & \\
$(\mathrm{n}=31)$ & & & \\
$\mathrm{t}$ & 18.9945 & 15.0686 & 22.9162 \\
$P$ & 0.0000 & 0.0000 & 0.0000 \\
\hline
\end{tabular}

\section{Discussion}

Gallbladder is the organ that concentrates and stores bile, which consists of four parts: bottom, body, neck and tube. The weakening of the gallbladder indicates diseases of the organ, which could affect the life of the patient without immediate intervention. Common digestive system diseases such as gallstones and polyp of gallbladder are all induced by gallbladder diseases, among which gallstones are induced by gallstones or bile ducts. Repeated stimulation of gallstones can cause inflammation and biliary obstruction, followed by abdominal pain, jaundice, vomiting and other symptoms. In severe cases, septic shock may occur, endangering people's lives. Gallbladder polyp is a disease that starts from the gallbladder wall and protrudes or bulges into the gallbladder cavity. It has no specific symptoms, and is accompanied by abdominal discomfort. Without timely intervention, it can progress to malignancy, increasing the difficulty of treatment. With changes of people's life and diet, the incidence of digestive diseases has gradually increased in recent years, including gallstones and gallbladder polyps. It has been reported that the single occurrence of gallstones and gallbladder polyps is easy to handle, with about $60 \%$ of them requiring no surgery. Once gallstones are combined with gallbladder polyps, surgery must be carried out immediately to avoid aggravation or even death.

Although cholelithiasis and gallbladder polyp can be removed by conventional laparotomy, recovery after surgery has proved difficult because of the long incision and great trauma, which could induce complications such as fever and vomiting. Secondly, intraoperative surgical instruments could damage the hepatic bile ducts, which in turn affects the digestive function of patients and slows down the recovery. Laparoscopic surgery is a new minimally invasive surgery in recent years, which can not only overcome the limitations of conventional open surgery, but also improve its prognosis. Moreover, literatures show that laparoscopic surgery is more effective and safer than open surgery.

By comparison, the clinical effect of the observation 
group was $96.77 \%$, higher than that of the control group $(80.65 \%)$, while the complication rate was $3.22 \%$, lower than that of the control group (22.58\%), which confirmed that laparoscopic cholecystectomy was a safe and effective treatment of gallstones with gallbladder polyps. There are several reasons behind it. Firstly, laparoscopic cholecystectomy is directly operated under laparoscopy, and doctors do not need to separate the triangular area of gallbladder, causing fewer damages to the body. Furthermore, the intraoperative physician can accurately separate the abdominal cavity structure so as to avoid damages to bile duct and liver caused by surgical instruments, thus better securing the patients during surgery.

The observation group was found to have a lower level of pain than that of the control group at 12 hours (3.64 \pm 0.52 points), 24 hours $(2.84 \pm 0.34$ points $)$ and 48 hours $(2.02 \pm 0.14$ points) after the surgery, confirming the role of laparoscopic cholecystectomy in alleviating the pain for patients. Analysis: Four $0.5-1.0 \mathrm{~cm}$ incisions were performed in the laparoscopic surgery, and the wound was smaller than that of open surgery, thus accelerating the healing process. Moreover, only band-aid was used to paste the wound, without the need of suture, thus lowering the level of pain. In addition, as laparoscopic cholecystectomy was directly performed under laparoscopy, it not only improved the accuracy of operation, but also avoided accidental injury of surrounding tissues by surgical instruments during operation, further alleviating the pain.

Compared with the control group, indicators of the observation group, including the operation duration (38.64 $\pm 14.42 \mathrm{~min})$, blood loss $(30.42 \pm 8.21 \mathrm{ml})$, length of stay $(4.71 \pm 1.82 \mathrm{~d})$, first anal exhaust $(21.82 \pm 6.65 \mathrm{~min})$, drainage volume $(72.02 \pm 4.21 \mathrm{ml})$, length of incision $(1.22 \pm 0.21 \mathrm{~cm})$ and the time for the recovery of gastrointestinal functions $(18.52 \pm 4.62 \mathrm{~h})$ were significantly better $(\mathrm{P}<0.05)$, as shown in Table 1 . It proved that laparoscopic cholecystectomy could significantly improve the efficacy of the surgery. Analysis: (1) In the laparoscopic cholecystectomy, a catheter was inserted deep into the abdominal cavity, followed by the injection of certain amount of $\mathrm{CO} 2$ to keep the pressure of the abdominal cavity at the level of $12-14 \mathrm{mmHg}$. Then four small holes were cut in the abdomen, where the operation was performed under the guidance of laparoscope. The surgery usually lasted for 40-90min because all the operations were carried out under the guidance of laparoscope, which can not only accurately locate the focus, but also shorten the time needed for locating the focus. Also, incisions caused by laparoscopic cholecystectomy tended to be small, which could avoid unnecessary tissue damages under the guidance of laparoscopy. Therefore, the loss of blood during operation was usually less than that of the control group. (2) Intravenous anesthesia is often used in laparoscopic cholecystectomy. Patients can get out of bed $7 \mathrm{~h}$ after the surgery, eat in 14-24 hours, and be discharged from hospital in 3-5 days. In addition, this surgery produces little trauma to the body, allowing the incision to heal completely about 1 week after operation.
Also, the gastrointestinal function of patients will return to normal. So, it is not easy to have complications such as infections during hospitalization and recovery after surgery.

In conclusion, laparoscopic cholecystectomy is an effective treatment of gallstones with gallbladder polyps, which can shorten operation duration and length of stay, and reduce the level of pain and complication rates. However, there were few literatures to refer to. Also, the size of samples was limited, and there was a lack of analysis of the immune status and the level of inflammatory factors before and after the operation. Therefore, future studies need to expand the size of samples and references. By comparing indicators of immunity and inflammations, we could determine the specific value of laparoscopic treatment, providing references for clinicians in treating the disease.

\section{References}

1. Pi Ruxian, Long Yuping, Fan Huiling, et al. Safety and Prognosis of Laparoscopic Cholecystectomy for Acute Cholecystitis Complicated with Gallstones [J]. Chongqing Medical Journal, 2018, 47 (002): 198-199, 202.

2. Yang Wen, Long Hao. Clinical Analysis of Early Laparoscopic Cholecystectomy for Cholecystolithiasis with Mild Acute Pancreatitis [J]. Chongqing Medical Journal, 2018,47 (019): 2624-2626.

3.Xu Guoliang, Wang Dongshu, Zhang Guoping, et al. Clinical Study of Compound Azintamide Enteric-coated Tablets Combined with Lansoprazole Enteric-coated Tablets and Domperidone Tablets in Patients with Gallstones after Laparoscopic Cholecystectomy [J]. Chinese Journal of Clinical Pharmacology, 2020,36 (11): 58-60.

4.Sun Peiyan, Han Mingyang, Wu Gang, et al. Clinical Analysis of 69 Cases of Laparoscopic Radical Gastrectomy Combined with Cholecystectomy [J]. Guangdong Medical Journal, 2018, 039 (0z1): 82-83. 